\title{
Ultrashort-laser electron-hole plasma and intragap states in diamond
}

\author{
G. K. Krasin ${ }^{1, a} \mathbb{C D}_{0}$, S. I. Kudryashov ${ }^{1}$, P. A. Danilov ${ }^{1}$, N. A. Smirnov ${ }^{1}$, A. O. Levchenko ${ }^{1}$, and M. S. Kovalev ${ }^{1,2}$ \\ ${ }^{1}$ Lebedev Physical Institute, Moscow, Russia 119991 \\ 2 Bauman Moscow State Technical University, Moscow, Russia 105005
}

Received 25 April 2021 / Accepted 22 July 2021 / Published online 9 August 2021

(C) The Author(s) 2021, corrected publication 2021

\begin{abstract}
The article discusses the implementation of ultrafast broadband excitation of A-band luminescence of natural diamond by femtosecond-laser pulses with low-energy near-IR photons. Such regime provides A-band luminescence yield with the fourth-power nonlinearity at low laser intensities and secondpower nonlinearity at higher laser intensities. These nonlinear dependences of A-band intensity on laser intensity can be related to quasi-resonant four-photon excitation at lower laser intensities and impact excitation of corresponding optical centers at higher intensities, or to similar nonlinear processes in electron-hole plasma relaxing to trapping donor-acceptor centers.
\end{abstract}

\section{Introduction}

The strict periodicity in the arrangement of the particles (atoms, molecules, ions) constituting the substance is a distinctive feature of the crystalline state. Any violation of this periodicity and balance leads to an appearance of a defect. Since impurities and defects change the properties of crystals, including diamonds, their study is therefore very important [1]. For example, diamond optical centers as optically active point defects have very long times of quantum coherence, which opens up wide possibilities for their practical application in various quantum technologies [2-4]. The use of such centers as optical probes for the local measurement of magnetic, electric and thermal fields or as photoluminescent biomarkers is also of great interest $[5,6]$.

To date, mainly point centers which are optically active in different regions of the visible and near infrared range are considered. Such point defects are denoted as $\mathrm{X}-\mathrm{V}$ centers, where $\mathrm{X}$ is an atom of nitrogen $(\mathrm{N})$, silicon $(\mathrm{Si})$, germanium $(\mathrm{Ge})$, phosphorus $(\mathrm{P})$, boron (B), titanium ( $\mathrm{Ti}$ ), etc., and $\mathrm{V}$ is a vacancy of the crystal structure of diamond. The substitutional atom and vacancy are associated with quasi-local lattice vibrations around the centers [7]. Such vibrations manifest themselves in the form of phonon repetitions of the zero-phonon line (ZPL) of the electronic transition between the excited and ground state of the center in the absorption (anti-Stokes lines) and luminescence (Stokes lines) spectra. As a result, they determine depolarization, nonradiative relaxation (quenching), and spin-orbit interaction for the excited state of the center, that is, polarization, spin characteristics,

\footnotetext{
${ }^{\mathrm{a}}$ e-mail: krasin.georg@gmail.com (corresponding author)
}

line width, yield and kinetics of optical emission as the most important optical characteristics of a quantum emitter.

Usually highly sensitive photoluminescence (PL) method $[8,9]$ is used to study the vibrational spectrum of diamond optical centers, one of the advantages of which is a fairly wide spectrum of excited emission. However, there is a mandatory overexcitation of the material with thermal quenching of the PL and excitation of the entire vibrational spectrum, which makes the observation of vibronic repetitions of ZPL for individual (especially low-energy, up to 20-30 meV [8]) modes impossible. It can be partially solved by preliminary cooling of the sample to helium temperatures.

At the same time, the possibility of resonant twophoton excitation of PL by ultrashort laser pulses of the NIR range was recently demonstrated for an elementary $\mathrm{N}-\mathrm{V}$ center in artificial diamond [10]. A similar multiphoton excitation of optical centers within the bandgap by highly penetrating ultrashort pulses in the visible and NIR ranges with varying degrees of nonlinearity of the photoionization allows their broadband-through the entire bandgap up to the conduction band-quasiresonant photoexcitation near the ZPL, characterized by high efficiency and minimal overexcitation in the spectral region under study. An interesting variation of this method is ultrafast laser heating of photoionized free carriers, the kinetic energy spectrum of which can be used to excite optical centers inside the bandgap by highly efficient resonant capture. So far, that mechanism has been considered only for laser-induced impact ionization of electrons in the valence band by highenergy electrons [11], however, never before for the excitation of interband electronic phenomena. Finally, there is also a promising method for time-resolved spectral 

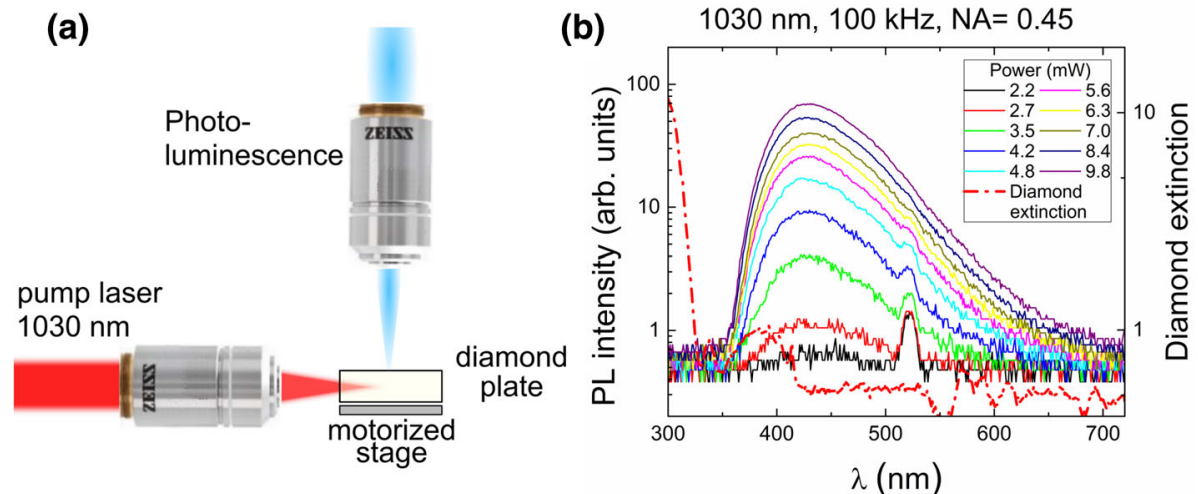

Fig. 1 The experimental layout (a) and the general view of PL spectrum of the diamond (b)

polarization studies of ultrafast PL, when the vibronic structure of optical centers manifests itself in the temporal modulation of the polarization-sensitive PL intensity and can be determined by Fourier analysis of the PL kinetics.

Thus, in order to expand the spectral range of emission of diamond optical centers that are promising for nanophotonics and quantum optics to the entire visible and NIR region, it is still necessary to thoroughly study their structural and energy characteristics, which determine the internal nonradiative relaxation processes in optical phenomena. In this paper, we study broadband luminescence spectrum from natural diamond rich in A-band excited by NIR ultrashort laser pulses.

\section{Experimental details}

The multi-photon interband photoexcitation of the nitrogen-doped (>700 ppm) IaA/B-type diamond (direct bandgap: $6.5 \mathrm{eV}$ [12], dimensions - $221 \mathrm{~mm}^{3}$ ) was observed to occur well above its absorption cutoff wavenumber of $\approx 30,000 \mathrm{~cm}^{-1}$ and generate electronhole plasma (EHP). A train of pulses from a $\mathrm{Yb}$ doped fiber laser (Satsuma, Amplitude Systemes) at $100 \mathrm{kHz}$ frequency, with $300 \mathrm{fs}$ widths at a wavelength of $1030 \mathrm{~nm}$ and different pulse energies $E=20-100$ nJ, was focused using a 0.45 numerical aperture (NA) micro-objective lens (Nikon) onto a focal spot of diameter $2 \mu \mathrm{m}\left(1 / \mathrm{e}^{2}\right.$ intensity level $)$ inside the diamond through its polished side windows (Fig. 1a); the resulting room-temperature photoluminescence (PL) was collected using a $0.2 \mathrm{NA}$ objective and then focused as a luminous track onto an input slit of a broadband spectrometer (ASP-150F).

\section{Experimental results and discussion}

The study was focused on the dependence of the PL yield on the peak intensity of incident ultrashort pulses, potentially indicating their mechanism of photoexcitation through their "cold" quasi-resonant four-photon excitation in one case and impact excitation with a higher luminescence yield in another one. In fact, the obtained experimental intensity-dependent PL yield showed a nonlinear character for all spectrally resolved bands with quasi-linear character for both low and medium ultrafast pulses intensity regions. The study of $\mathrm{PL}$ was carried out in pre-filamentation regime, where self-focusing power for diamond in the near IR was taken equal to $P_{\text {cr.diam }} \approx 0.5 \mathrm{MW}$ for near-IR pulses $[13,14]$ (for comparison, the range of ultrashort pulses peak power was $P=50-350 \mathrm{~kW}$ ).

The kinetic rate equation $[15,16]$ has proved to be a simple and informative approach to the analysis of the electron-hole plasma (EHP) dynamics in dielectrics excited by ultrashort pulses. Given the fundamental multi-photon interband absorption of type "valence band-conduction band" of diamond, the kinetic rate equation for the EHP density $\rho_{e, h}$ can be written in the form [17]

$$
\frac{\mathrm{d} \rho_{e, h}}{\mathrm{~d} t}=\sigma_{n} I^{n}+\alpha I \rho_{e, h}-\beta \rho_{e, h}^{2}-\frac{\rho_{e, h}}{\tau_{S T}}
$$

where the first term on the right side reflects multiphoton absorption; the second determines impact ionization; the third and fourth ones correspond to threebody Auger and radiative two-body recombination, respectively, and the last one is the trapping of free carriers with a characteristic time constant $\tau_{S T}$. In this equation, the radiation-recombination contribution of the integral form corresponds to the PL yield

$$
\Phi \propto \int \beta \rho_{e, h}^{2} \mathrm{~d} t
$$

Since the key coefficients of Eq. (1) for diamond are usually unknown, it is of interest to analyze the equation in general in comparison with the PL yield according to Eq. (2).

In this particular work, at low intensities of ultrashort pulses multi-photon excitation of A-band-related centers with the concentration $\rho_{A}$ could occur 


\section{(a)}

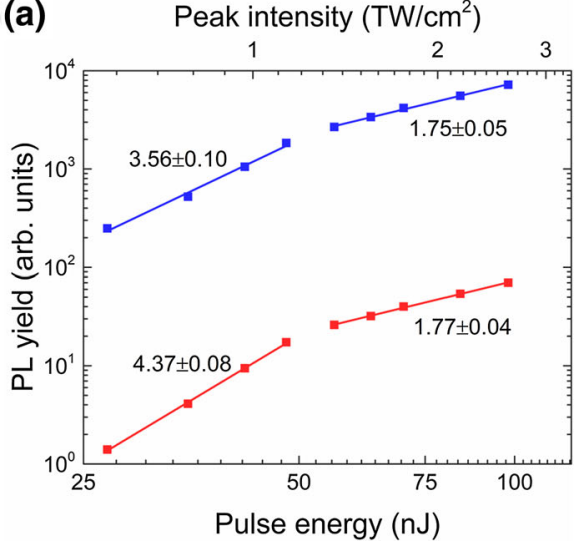

(b)
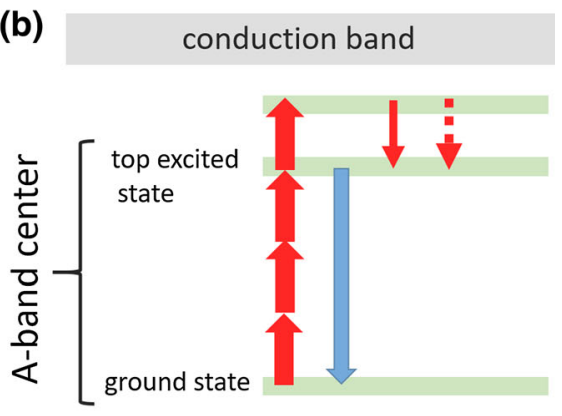

valence band

Fig. 2 Dependences of PL yield on the ultrashort pulses energy for peak intensities of the spectra (red) and for spectrally integrated intensity (blue), as well as their linear interpolations showing the power slopes of the dependences (a). Diagram of processes associated with direct multiphoton photoexcitation of EHP and relaxation (red) in the region of the main bands and accompanying photoluminescence (blue) in diamond (b)

$$
\begin{aligned}
\frac{\mathrm{d} \rho_{e, h}}{\mathrm{~d} t}= & \sigma_{4} I^{4} \rho_{A}-\beta \rho_{e, h}^{2}, \quad \sigma_{4} I^{4} \rho_{A} \approx \beta \rho_{e, h}^{2}, \\
& \rho_{e, h} \propto I^{2}
\end{aligned}
$$

resulting in the PL yield in the form

$$
\Phi \propto \int \beta \rho_{e, h}^{2} \mathrm{~d} t \propto I^{4}
$$

demonstrating the same nonlinearity of the intensitydependent luminescence yield as the observed in Fig. 2a.

Then, at medium intensities of ultrashort pulses, predominating impact ionization could provide the same excitation path. In this case, the kinetic equation and the corresponding PL yield can be written in the form

$$
\begin{aligned}
& \frac{\mathrm{d} \rho_{e, h}}{\mathrm{~d} t}=\alpha \rho_{e, h} I \rho_{A}-\beta \rho_{e, h}^{2}, \quad \alpha \rho_{e, h} I \rho_{A} \approx \beta \rho_{e, h}^{2}, \\
& \rho_{e, h} \propto I^{1} \\
& \Phi \propto \int \beta \rho_{e, h}^{2} \mathrm{~d} t \propto I^{2}
\end{aligned}
$$

reflecting a much slower rise in the PL yield from the pulse intensity. As it is obvious from the qualitatively similar PL spectra (Fig. 1b), the change in the nonlinearities (slopes of the linear fitting) in Fig. 2a, does not change the PL mechanism. Hence, one can suggest in both these cases, the electrons promoted from the Aband center to the conduction band, could return to the A-band center excited state radiatively (NIR range) or non-radiatively, ending up with the observed blue-range photoluminescence.

Alternatively, the same nonlinearities of A-band PL yield versus 1030-nm fs-laser intensity can be observed during the continuous set of transition regimes, gradually turning on additional strong effects in dynamics of electron-hole plasma with its eventually increasing density - density-limiting Auger recombination, lowpower impact ionization mechanism, etc., in Eq. (1). In such case, the A-band PL yield nonlinearity could continuously decrease versus fs-laser intensity, as observed through comparison of the intensity-dependent nonlinearities in this and our recent work [18] at rather similar experimental conditions. Meanwhile, even in this situation the difference in focusing NA $=0.25[18]$ and 0.45 (this study) could introduce considerable difference in the density of excited carriers in their focal volumes and the resulting A-band photoluminescence yield through ultrafast lateral electron-hole plasma densitydependent ambipolar diffusion during the pumping 300fs laser pulses [19], which is not well-studied yet in diamond at high-excitation densities [20].

\section{Conclusion}

Ultrafast near infrared-range excitation of nitrogen-rich natural diamond produces mainly visible photoluminescence with nonlinear character of its yield as a function of laser intensity. The A-band photoexcitation at lower intensities of ultrashort pulses can be explained by multi-photon excitation of corresponding intra-gap centers occurring between the center populated ground state and conduction band. Then, for the higher intensities of ultrashort pulses the impact ionization of the center becomes dominating factor, which could provide the same excitation path. Alternatively, the same nonlinearities of A-band PL yield versus laser intensity can be explained via continuous set of transition regimes, gradually turning on different strong effects in dynamics of electron-hole plasma of increasing density.

Acknowledgements This research was funded by Russian Science Foundation, Grant No. 21-79-30063. 


\section{Author contributions}

All authors contributed equally to the paper.

Data Availability Statement This manuscript has no associated data or the data will not be deposited. [Authors' comment: Data underlying the results presented in this manuscript are available from the authors upon reasonable request.].

Open Access This article is licensed under a Creative Commons Attribution 4.0 International License, which permits use, sharing, adaptation, distribution and reproduction in any medium or format, as long as you give appropriate credit to the original author(s) and the source, provide a link to the Creative Commons licence, and indicate if changes were made. The images or other third party material in this article are included in the article's Creative Commons licence, unless indicated otherwise in a credit line to the material. If material is not included in the article's Creative Commons licence and your intended use is not permitted by statutory regulation or exceeds the permitted use, you will need to obtain permission directly from the copyright holder. To view a copy of this licence, visit http://creativecomm ons.org/licenses/by/4.0/.

\section{References}

1. L. Ondič, M. Varga, J. Fait, K. Hruška, V. Jurka, A. Kromka, J. Maňák, P. Kapusta, J. Nováková, Nanoscale 12, 13055 (2020). https://doi.org/10.1039/ C9NR10580H

2. Y.-C. Chen, B. Griffiths, L. Weng, S.S. Nicley, S.N. Ishmael, Y. Lekhai, S. Johnson, C.J. Stephen, B.L. Green, G.W. Morley, M.E. Newton, M.J. Booth, P.S. Salter, J.M. Smith, Optica 6, 662 (2019). https://doi.org/10. 1364/OPTICA.6.000662

3. D.D. Awschalom, R. Hanson, J. Wrachtrup, B.B. Zhou, Nat. Photon. 12, 516 (2018). https://doi.org/10.1038/ s41566-018-0232-2

4. J.P. Hadden, V. Bharadwaj, B. Sotillo, S. Rampini, R. Osellame, J.D. Witmer, H. Jayakumar, T.T. Fernandez, A. Chiappini, C. Armellini, M. Ferrari, Opt. Lett. 43, 3586 (2018). https://doi.org/10.1364/OL.43.003586

5. S. Blakley, X. Liu, I. Fedotov, I. Cojocaru, C. Vincent, M. Alkahtani, J. Becker, M. Kieschnick, T. Lühman, J. Meijer, P. Hemmer, ACS Photon. 6, 1690 (2019). https://doi.org/10.1021/acsphotonics.9b00206
6. J.M. Taylor, P. Cappellaro, L. Childress, L. Jiang, D. Budker, P.R. Hemmer, A. Yacoby, R. Walsworth, M.D. Lukin, Nat. Phys. 4, 810 (2008). https://doi.org/10. 1038/nphys 1075

7. A.M. Zaitsev, Optical Properties of Diamond: A Data Handbook (Springer, Berlin, 2003)

8. G. Davies, Rep. Prog. Phys. 44, 787 (1981). https:// doi.org/10.1088/0034-4885/44/7/003

9. H.-C. Lu, M.-Y. Lin, S.-L. Chou, Y.-C. Peng, J.-I. Lo, B.-M. Cheng, Anal. Chem. 84, 9596 (2012). https://doi. org/10.1021/ac302545u

10. S.I. Kudryashov, R.A. Khmelnitskii, P.A. Danilov, N.A. Smirnov, A.O. Levchenko, O.E. Kovalchuk, M.V. Uspenskaya, E.A. Oleynichuk, M.S. Kovalev, Opt. Lett. 46, 1438 (2021). https://doi.org/10.1364/OL.414583

11. S.S. Mao, F. Quéré, S. Guizard, X. Mao, R.E. Russo, G. Petite, P. Martin, Appl. Phys. A 79, 1695 (2004). https://doi.org/10.1007/s00339-004-2684-0

12. E.D. Palik, In Handbook of Optical Constants of Solids (Academic Press, New York, 1998)

13. S.I. Kudryashov, A.O. Levchenko, P.A. Danilov, N.A. Smirnov, A.A. Ionin, Opt. Lett. 45, 2026-2029 (2020). https://doi.org/10.1364/OL.389348

14. P. Liu, R. Yen, N. Bloembergen, IEEE J. Quantum Electron. 14, 574 (1978). https://doi.org/10.1109/JQE. 1978.1069857

15. M. Mero, J. Liu, W. Rudolph, D. Ristau, K. Starke, Phys. Rev. B 71, 115109 (2005). https://doi.org/10. 1103/PhysRevB.71.115109

16. S. Kudryashov, P. Danilov, A. Rupasov, S. Khonina, A. Nalimov, A. Ionin, G. Krasin, M. Kovalev, Opt. Mater. Express 10, 3291 (2020). https://doi.org/10. 1364/OME.412399

17. S.I. Kudryashov, A.O. Levchenko, P.A. Danilov, N.A. Smirnov, A.E. Rupasov, R.A. Khmelnitskii, O.E. Kovalchuk, A.A. Ionin, JETP Lett. 112, 533 (2020). https://doi.org/10.1134/S0021364020210080

18. S. Kudryashov, N. Stsepuro, P. Danilov, N. Smirnov, A. Levchenko, M. Kovalev, Opt. Mater. Express 11, 22342241 (2021). https://doi.org/10.1364/OME.428640

19. N.A. Smirnov, S.I. Kudryashov, A.A. Rudenko, D.A. Zayarny, A.A. Ionin, Appl. Surf. Sci. 562, 150243 (2021). https://doi.org/10.1016/j.apsusc.2021.150243

20. M. Kozák, F. Trojánek, P. Malý, New J. Phys. 17, 053027 (2015). https://doi.org/10.1088/1367-2630/17/ $5 / 053027$ 